\title{
Tuberculous Lymphadenitis Mimicking Gastric Subepithelial Tumor Diagnosed Using Endoscopic Ultrasound-guided Fine-needle Aspiration
}

\author{
Sung Bum Kim, Tae Nyeun Kim, Kook Hyun Kim \\ Division of Gastroenterology and Hepatology, Department of Internal Medicine, Yeungnam University College of Medicine, Daegu, Korea
}

\begin{abstract}
A gastric subepithelial tumor (SET) is commonly detected during a diagnostic endoscopic examination. Gastric tuberculosis (TB), in particular, can present as a SET of the gastric wall. A few cases of gastric TB mimicking a SET have recently been reported. Radiological imaging combined with endoscopic biopsy can aid in the early diagnosis of TB without surgical intervention. A 41-year-old man visited our health promotion center for a regular check-up. Esophagogastroduodenoscopy (EGD) revealed a round and smooth, bulging mucosal lesion suggesting a gastric SET in the upper body of the stomach. Endoscopic ultrasound (EUS) demonstrated a hypoechoic lesion measuring $18 \mathrm{~mm}$, with an obscure layer of origin, and EUS-guided fine-needle aspiration was performed. Microscopic examination of the sample showed chronic granulomatous inflammation. Histopathologically, the aspirated sample showed positive Ziehl-Neelsen staining, confirming a diagnosis of tuberculous lymphadenitis. We describe a case of a patient who presented with tuberculous lymphadenitis mimicking a SET of the stomach. The lesion was found on EGD and confirmed using EUS-guided fine-needle aspiration. (Korean J Helicobacter Up Gastrointest Res 2018;18:65-69)
\end{abstract}

Key Words: Endoscopic ultrasound; Endoscopic ultrasound-guided fine-needle aspiration; Subepithelial tumor; Tuberculosis

\section{INTRODUCTION}

Tuberculosis (TB) is an important economic burden and health problem globally. ${ }^{1}$ Although TB can involve multiple organs, the lungs are known to be most commonly affected, followed by the gastrointestinal and genitourinary tracts. Abdominal TB, which comprises approximately $1 \sim 3 \%$ of all cases of $\mathrm{TB}$, is an extrapulmonary manifestation that usually originates from a primary focus of pulmonary $\mathrm{TB}^{2,3}$ Tuberculous involvement of the stomach can present as a subepithelial tumor (SET). However, diagnosis of gastric TB is difficult because it requires a high index of clinical suspicion and an appropriate and adequate histopathological analysis. Few cases of gastric TB mimicking a SET have been reported in previous reports. ${ }^{4,5}$ We present a case of an asymptomatic patient who presented with tuberculous lymphadenitis resembling

Received: September 30, 2017 Revised: November 28, 2017 Accepted: November 29, 2017 Corresponding author: Kook Hyun Kim

Division of Gastroenterology and Hepatology, Department of Internal Medicine, Yeungnam University College of Medicine, 170 Hyeonchung-ro, Nam-gu, Daegu 42415, Korea

Tel: +82-53-620-3576, Fax: +82-53-654-8386, E-mail: kimkh@yu.ac.kr a SET of the stomach noted on esophagogastroduodenoscopic (EGD) examination, which was confirmed using endoscopic ultrasound-guided fine-needle aspiration (EUS-FNA).

\section{CASE REPORT}

A 41-year-old man, non-smoker, visited our health promotion center for a regular check-up. He was asymptomatic and demonstrated no significant findings on physical examination. He reported past history of pulmonary TB over 20 years prior to presentation, without any recurrence since. An EGD examination showed a well-defined bulging mucosal lesion measuring approximately 20 $\mathrm{mm}$ in size with an intact mucosal surface, suggesting the presence of a gastric SET, which was incidentally visualized on the anterior aspect of the upper body of the stomach (Fig. 1A). The lesion showed a smooth surface and firm consistency when probed with a biopsy forcep. However, an EGD examination 2 years prior to the present evaluation had been normal without a SET detected at the time of that evaluation (Fig. 1B), and a chest X-ray

Copyright $\odot 2018$ Korean College of Helicobacter and Upper Gastrointestinal Research

@) The Korean Journal of Helicobacter and Upper Gastrointestinal Research is an Open-Access Journal. All articles are distributed under the terms of the Creative Commons Attribution Non-Commercial License (http://creativecommons.org/licenses/by-nc/4.0) which permits unrestricted non-commercial use, distribution, and reproduction in any medium, provided the original work is properly cited. 

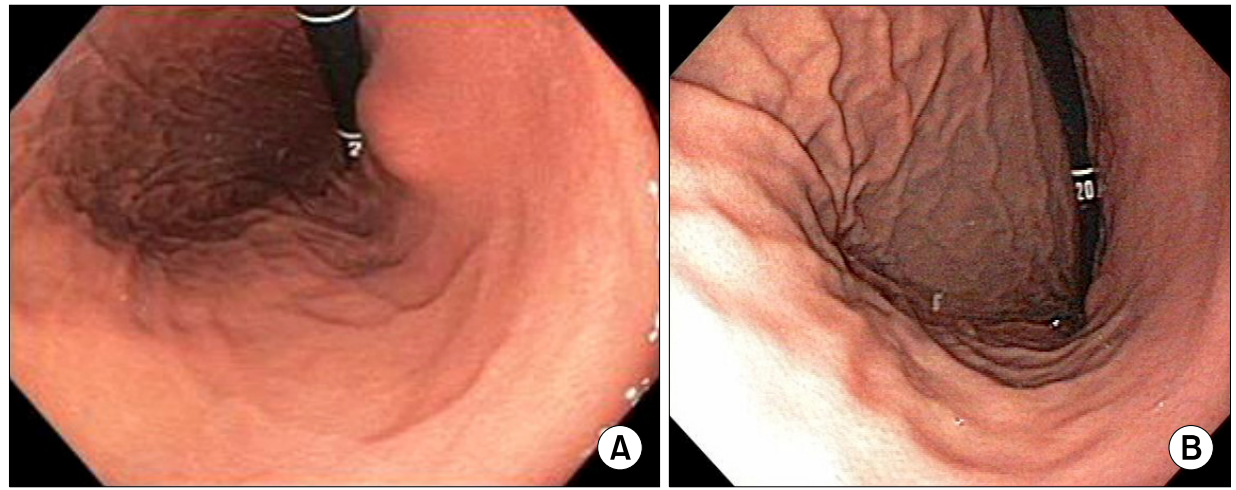

Fig. 1. (A) Esophagogastroduodenoscopy shows a smooth, elevated subepithelial tumor in the upper body of the stomach. (B) Esophagogastroduodenoscopy performed 2 years prior shows no subepithelial tumor in the upper body of the stomach.

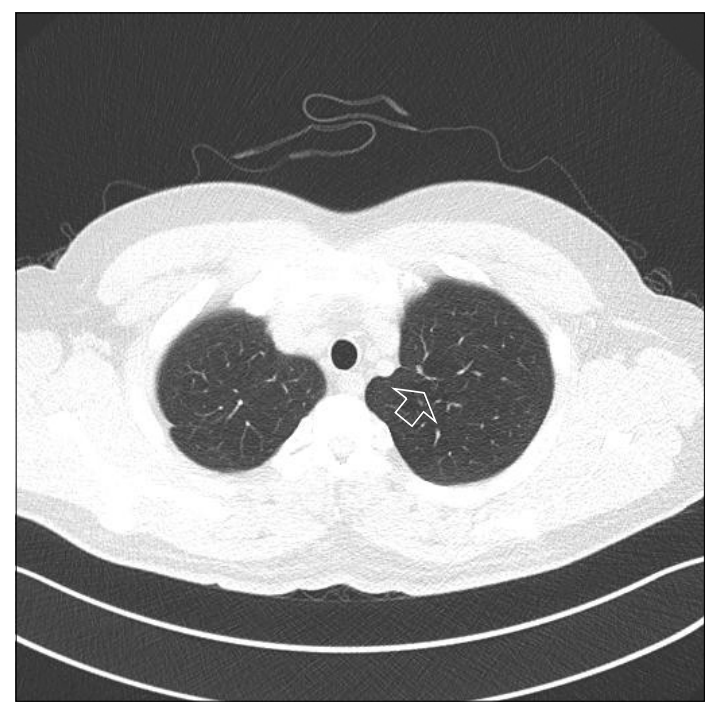

Fig. 2. Chest computed tomography shows focal scarring with small granulomas in bilateral upper and right lower lung lobes (arrow).

was unremarkable. Chest computed tomography (CT) showed focal scarring with small granulomas in both upper lobes and the right lower lobe of the lungs (Fig. 2). Abdominal CT demonstrated a round, low-density lesion with peripheral calcification that was adherent to the stomach wall, and it was presumed to be a perigastric lymph node (Fig. 3). Upon admission, liver function tests and a blood chemistry panel showed: aspartate aminotransferase $31 \mathrm{IU} / \mathrm{L}$, alanine aminotransferase $13 \mathrm{IU} / \mathrm{L}$, white blood cell count 4,600 cells $/ \mathrm{mm}^{3}$ (reference range, 3,000 9,000 cells $/ \mathrm{mm}^{3}$ ), erythrocyte sedimentation rate 9 $\mathrm{mm} / \mathrm{h}$ (reference range, $0 \sim 20 \mathrm{~mm} / \mathrm{h}$ ), and C-reactive protein $0.2 \mathrm{mg} / \mathrm{dL}$ (reference range, $0 \sim 0.5 \mathrm{mg} / \mathrm{dL}$ ).

Radial EUS showed an 18-mm heterogeneously echoic round lesion with focal calcifications in the stomach, in-

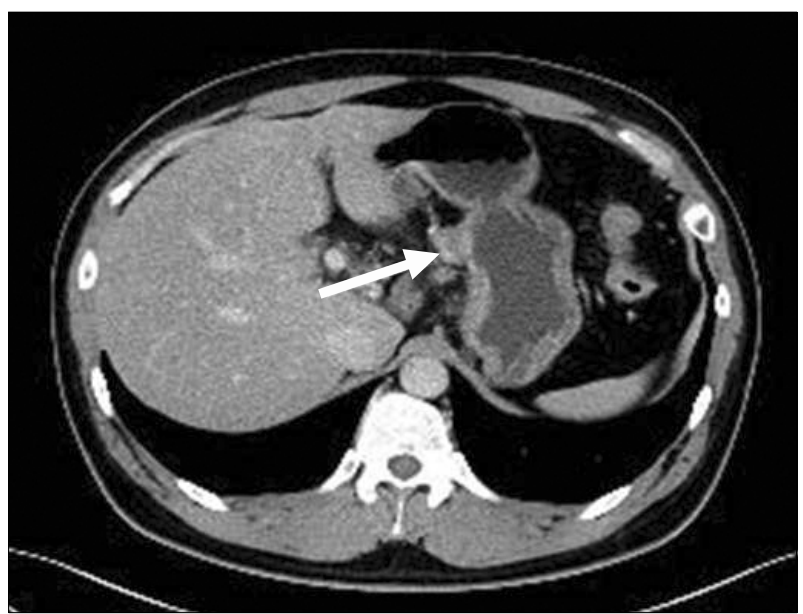

Fig. 3. Abdominal computed tomography demonstrates a round low-density lesion abutting the stomach, measuring approximately $20 \mathrm{~mm}$ in size (white arrow), with peripheral calcification.

distinguishable from the layers of the gastric wall (Fig. 4). An EUS-FNA was performed with a curved linear array scope (GF-UCT260; Olympus, ToKyo, Japan) using a 22-gauge needle (Expect 22G Flex; Boston Scientific, Natick, MA, USA) via a transgastric route. We performed 3 passes with a to-and-fro motion technique to ensure maximal aspiration of tissue. Following microscopic examination of the aspirated sample using the hematoxylin and eosin stain, we diagnosed the lesion as chronic granulomatous inflammation (CGI). Subsequently, we examined the sample using a Ziehl-Neelsen stain and a polymerase chain reaction for Mycobacterium tuberculosis to establish a differential diagnoses for CGI (Fig. 5). Ziehl-Neelsen staining showed positive results, and a diagnosis of TB was confirmed. A colonoscopic examination was performed to investigate and rule out extrapulmonary 

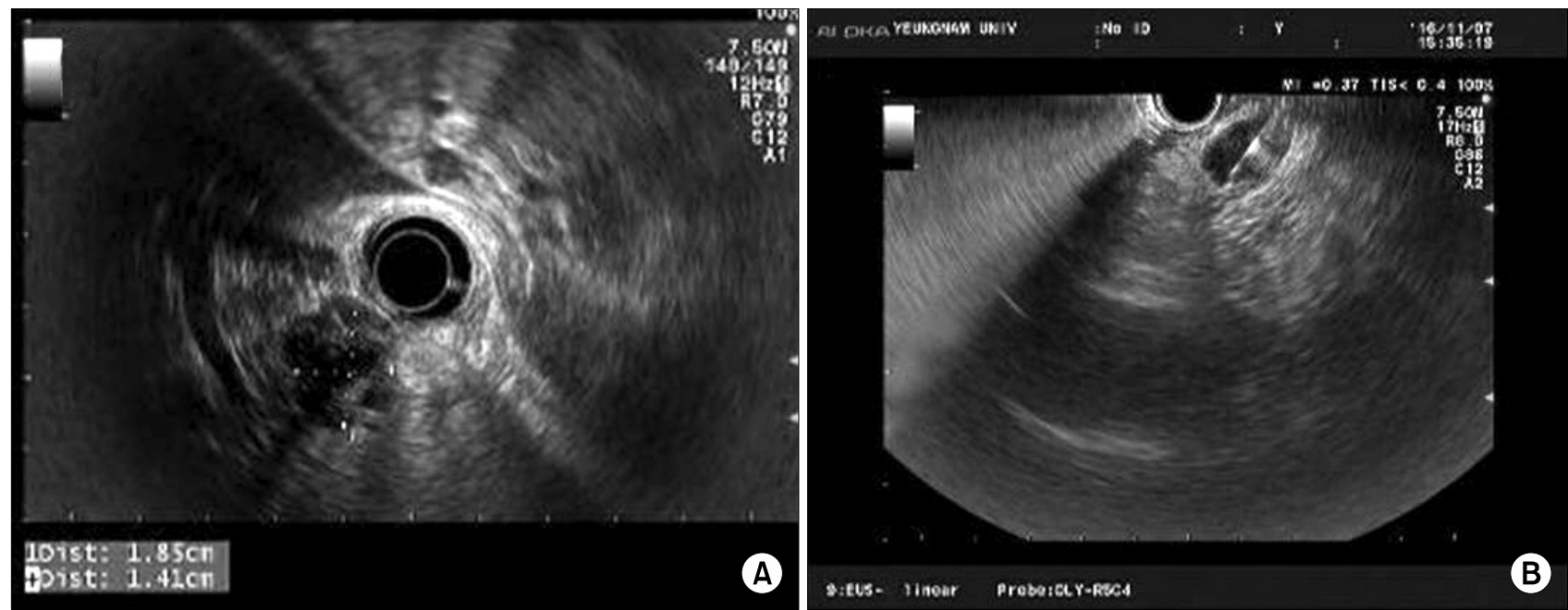

Fig. 4. (A) Radial endoscopic ultrasound shows an 18-mm hypoechoic, round mass indistinguishable from the layers of the gastric wall. (B) Endoscopic ultrasound-guided fine-needle aspiration of the mass is performed via a transgastric route by using a 22-gauge needle.

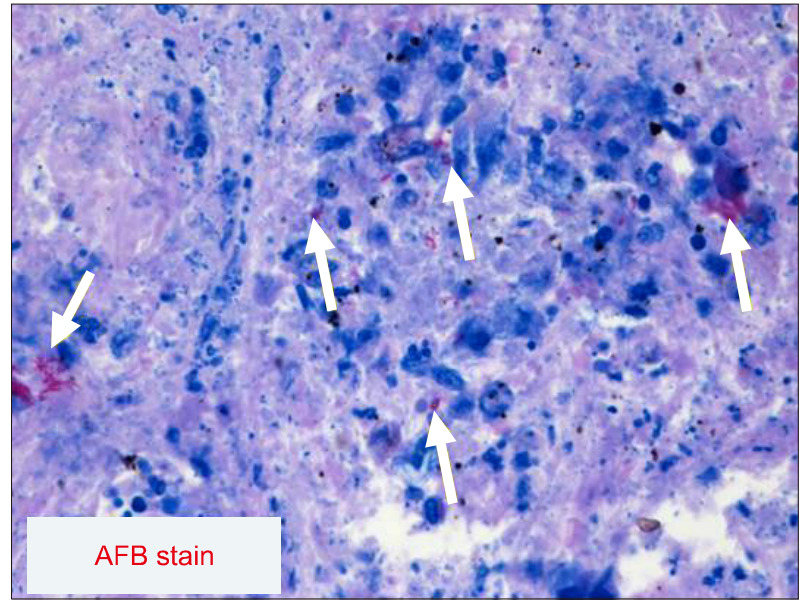

Fig. 5. Acid-fast bacilli (AFB) staining of the aspirated cells is positive, indicating the presence of Mycobacterium (arrows) (AFB stain, $\times 400)$.

TB. Colonoscopic examination showed a wide open ileocolonic valve and multiple scars in addition to a mucosal deformity around the cecum and the proximal ascending colon (Fig. 6).

Based on histopathological results, he was eventually diagnosed with tuberculous lymphadenitis and referred to a pulmonologist who initiated treatment with the standard drug regimen comprising 4 antituberculous drugs (isoniazid $400 \mathrm{mg}$, ethambutol $800 \mathrm{mg}$, rifampin $600 \mathrm{mg}$, and pyrazinamide $1,500 \mathrm{mg}$ ) orally for 2 months, followed by 3 drugs (isoniazid $400 \mathrm{mg}$, ethambutol $800 \mathrm{mg}$, and ri-

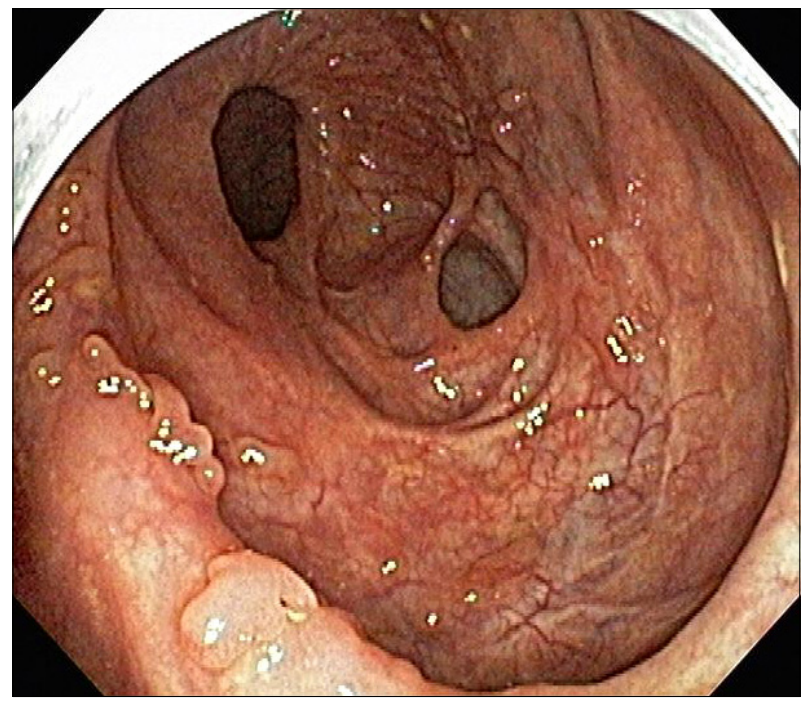

Fig. 6. Colonoscopy shows a wide-open ileocolonic valve and multiple scars in addition to a mucosal deformity around the cecum and proximal ascending colon.

fampin $600 \mathrm{mg}$ ) for 4 months. The patient did not report any adverse effects during medical treatment.

\section{DISCUSSION}

EUS is a well-established modality used for the evaluation of a SET of the gastrointestinal tract. EUS plays a key role in detailed evaluation to identify the layer of origin, the echo pattern, as well as the size, consistency and vas- 
cularity of a tumor. ${ }^{6}$ EUS can also differentiate between a SET and other factors causing extrinsic compression of the stomach by identifying the layer from which a SET originates and its relationship with the gastric wall. ${ }^{7}$ Extrinsic compression of the stomach can be caused by adjacent vascular structures, organs, or pathological structures such as masses, cysts, or lymphadenopathy. In this patient, bulging of the stomach resulted from extrinsic compression of the stomach caused by adjacent lymphadenopathy.

Abdominal TB can develop at any age; however, young adults aged between 25 and 45 years are most commonly affected. ${ }^{8}$ Although primary gastric TB is rare, the gastrointestinal tract is a common site of infection for $\mathrm{ex}^{-}$ trapulmonary $\mathrm{TB}^{4,9}$ The clinical presentation varies based on whether it is a primary infection or a lesion secondary to pulmonary TB. Combined radiological and endoscopic imaging can facilitate early diagnosis without the need to perform unnecessary surgical resection. A few case reports indicate that the presentation of $\mathrm{TB}$ gastritis or adenopathy can mimic SET-like lesions noted on EGD (Table 1). ${ }^{4,5,10-13}$ Previous EUS studies have revealed that in 4 of 6 cases studied the tumor was observed to have developed in the muscularis propria layer of the gastric wall, which is a common finding associated with gastrointestinal stromal tumors (GISTs). ${ }^{4,511,12}$ In 2 patients, the tumor was observed to be present outside the gastric wall- in 1 patient, it was visualized as a protruding mass with an overlying ulceration and in the other, presented as a SET-like lesion. ${ }^{10,13}$ Endosonographically, GISTs appear as well-circumscribed, hypoechoic round lesions located in the muscularis propria of the gastric wall. Gastric TB also can manifest as hypoechoic lesions developing within the muscularis propria, which makes gastric TB indistinguishable from GIST based on EUS findings. ${ }^{4}$

Histopathological assessment of SET is the key parameter that can aid in differentiating benign from malignant tumors and help to accurately diagnose structures causing extrinsic compression of the stomach. A meta-analysis evaluating the diagnostic efficacy of EUS-fine needle sampling for assessment of upper gastrointestinal subepithelial lesions showed moderate efficacy in providing a tissue diagnosis. ${ }^{14}$ Results of a tissue diagnosis of SET obtained after performing an EUS-FNA are occasionally inconclusive in patients suspected of presenting with tuberculous lymphadenitis; therefore, further evaluation with surgical resection or a repeat EUS-FNA should be considered.

Microscopic confirmation of TB remains a challenge. Arriving at a conclusive histopathological diagnosis of $\mathrm{TB}$ requires that samples of aspirated tissue or surgical specimens demonstrate caseating epitheloid granulomas or a positive result with the Ziehl-Neelsen stain. Of the $6 \mathrm{pa}^{-}$ tients reported in this context, only 2 were diagnosed as

Table 1. Summary of Tuberculous Gastropathy Mimicking a Subepithelial Tumor

\begin{tabular}{|c|c|c|c|c|c|c|c|c|}
\hline Author & $\begin{array}{l}\text { Sex/age } \\
\text { (yr) }\end{array}$ & Symptoms & EGD & EUS (origin/diameter) & $\begin{array}{c}\text { Tbc } \\
\text { history }\end{array}$ & $\begin{array}{l}\text { Diagnostic } \\
\text { tool }\end{array}$ & Pathology & Treatment \\
\hline $\begin{array}{l}\text { Kim et al. } \\
(2005)\end{array}$ & $\mathrm{F} / 43$ & Epigastric pain & $\begin{array}{l}\text { SET-like, } \\
\text { ulcer }\end{array}$ & $\begin{array}{l}\text { Outside of gastric } \\
\text { wall/no mention }\end{array}$ & Yes & EUS-FNA & $\operatorname{AFB}(+)$ & $\begin{array}{l}\text { Anti-TB } \\
\text { medication }\end{array}$ \\
\hline $\begin{array}{l}\text { Kim et al. } \\
(2005)\end{array}$ & $\mathrm{F} / 21$ & $\begin{array}{l}\text { Abdominal } \\
\text { discomfort }\end{array}$ & SET-like & MP layer/26 mm & No & Surgery & $\begin{array}{l}\text { TB-PCR }(+), \\
\text { caseation necrosis }\end{array}$ & $\begin{array}{l}\text { Anti-TB } \\
\text { medication }\end{array}$ \\
\hline $\begin{array}{l}\text { Niitsu et al. }{ }^{11} \\
(2012)\end{array}$ & $\mathrm{F} / 41$ & Incidental & SET-like & MP layer/23 mm & Yes & Surgery & Caseation necrosis & None \\
\hline $\begin{array}{l}\text { Gupta et al. } \\
(2012)\end{array}$ & $\mathrm{M} / 28$ & Epigastric pain & SET-like & MP layer/15 mm & No & Surgery & $\operatorname{AFB}(+)$ & $\begin{array}{l}\text { Anti-TB } \\
\text { medication }\end{array}$ \\
\hline $\begin{array}{l}\text { Lee et al. }{ }^{13} \\
(2013)\end{array}$ & $\mathrm{F} / 61$ & Incidental & SET-like & $\begin{array}{l}\text { Outside of gastric } \\
\text { wall } / 30 \mathrm{~mm}\end{array}$ & No & EUS-FNB & $\begin{array}{c}\operatorname{AFB}(+), \operatorname{TB}-\operatorname{PCR}(+), \\
\text { caseation necrosis }\end{array}$ & $\begin{array}{l}\text { Anti-TB } \\
\text { medication }\end{array}$ \\
\hline $\begin{array}{l}\text { Imbe et al. } \\
(2014)\end{array}$ & $\mathrm{M} / 71$ & Incidental & SET-like & MP layer/15 mm & No & EUS-FNA & CGI & None \\
\hline
\end{tabular}

EGD, esophagogastroduodenoscopy; EUS, endoscopic ultrasound; F, female; M, male; SET, subepithelial tumor; EUS-FNA, endoscopic ultrasound guided fine-needle aspiration; AFB, acid-fast bacilli; TB, tuberculosis; MP, muscularis propria; PCR, polymerase chain reaction; CGI, chronic granulomatous inflammation. 
having TB after performing an EUS-FNA.,10 One patient showed CGI on histopathological evaluation but a negative result with Ziehl-Neelsen staining. ${ }^{5}$ The others reported previous history of TB and demonstrated the presence of acid-fast bacilli in the aspirated cells - a finding similar to our case. ${ }^{10}$ Once EUS-FNA establishes the diagnosis of $\mathrm{TB}$, anti-TB chemotherapy is typically recommended as the treatment of choice. Fortunately, our patient did not require unnecessary surgery because the EUS-FNA procedure could conclusively confirm TB in tissue samples.

In conclusion, SET-like lesions are often detected during an endoscopy. EUS is a useful modality to aid in differentiating a SET from extraluminal masses and to identify the morphological features of the lesion. EUS-FNA is primarily recommended in patients to obtain a minimally invasive diagnosis without need of surgery. Both, detailed history taking and careful histopathological analysis are important to conclusively diagnose tuberculous lymphadenitis that mimics a SET in the stomach.

\section{REFERENCES}

1. World Health Organization. Global tuberculosis report, 2016. Geneva: WHO, 2016 [cited 2016 Nov 8]. Available from: http://apps.who.int/iris/bitstream/10665/250441/1/ 9789241565394-eng.pdf?ua=1

2. Arabi NA, Musaad AM, Ahmed EE, Ibnouf MM, Abdelaziz MS. Primary gastric tuberculosis presenting as gastric outlet obstruction: a case report and review of the literature. J Med Case Rep 2015;9:265.

3. Sheer TA, Coyle WJ. Gastrointestinal tuberculosis. Curr
Gastroenterol Rep 2003;5:273-278.

4. Kim SH, Park JH, Kang KH, et al. Gastric tuberculosis presenting as a submucosal tumor. Gastrointest Endosc 2005;61:319-322.

5. Imbe K, Irisawa A, Shibukawa G, et al. Idiopathic granulomatous gastritis diagnosed with endoscopic ultrasound-guided fine-needle aspiration: report of a case. Endosc Int Open 2014;2:E259-E261.

6. Papanikolaou IS, Triantafyllou K, Kourikou A, Rösch T. Endoscopic ultrasonography for gastric submucosal lesions. World J Gastrointest Endosc 2011;3:86-94.

7. Oztas E, Oguz D, Kurt M, et al. Endosonographic evaluation of patients with suspected extraluminal compression or subepithelial lesions during upper gastrointestinal endoscopy. Eur J Gastroenterol Hepatol 2011;23:586-592.

8. Akgun Y. Intestinal and peritoneal tuberculosis: changing trends over 10 years and a review of 80 patients. Can J Surg 2005; 48:131-136.

9. Mitchell RS, Bristol LJ. Intestinal tuberculosis: an analysis of 346 cases diagnosed by routine intestinal radiography on 5,529 admissions for pulmonary tuberculosis, 1924-49. Am J Med Sci 1954;227:241-249.

10. Kim DY, Bang S, Park BK, et al. Tuberculous mesenteric lymphadenitis involving the gastric wall: case report. Gastrointest Endosc 2005;62:799-802.

11. Niitsu H, Tanabe K, Tokumoto N, et al. Idiopathic granulomatous gastritis resembling a gastrointestinal stromal tumor. Case Rep Gastroenterol 2012;6:502-509.

12. Gupta V, Goel MM, Noushif M, Rai P, Gupta P, Chandra A. Primary gastric tuberculosis mimicking gastrointestinal stromal tumor. Am J Gastroenterol 2012;107:1269-1270.

13. Lee TH, Cho JY, Bok GH, Cho WY, Jin SY. Intra-abdominal tuberculous lymphadenitis diagnosed using an endoscopic ultrasonography-guided procore needle biopsy. Clin Endosc 2013;46:77-80.

14. Zhang XC, Li QL, Yu YF, et al. Diagnostic efficacy of endoscopic ultrasound-guided needle sampling for upper gastrointestinal subepithelial lesions: a meta-analysis. Surg Endosc 2016;30: 2431-2441. 